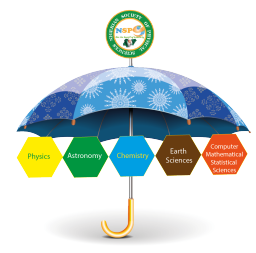

\title{
Eigenvalue Elasticity and Sensitivity Analyses of the Transmission Dynamic Model of Corruption
}

\author{
Adeyemi Olukayode Binuyo* \\ Department of Mathematical Sciences, Ajayi Crowther University, Oyo, Nigeria
}

\begin{abstract}
In this paper, the eigenvalue elasticity and sensitivity values of the mathematical model of transmission dynamics of corruption were obtained and presented using the eigenvalue elasticity and sensitivity analysis methods. The parameter with the greatest impact on the mathematical model was determined using the methods. This parameter will assist the government on how to reduce and provide measures to eradicate corrupt practices among the populace. From the mathematical model of corruption presented, it was obtained that the effective contact rate of corruption has the highest value when using the eigenvalue elasticity and sensitivity analysis.
\end{abstract}

Keywords: Corruption, Mathematical Model, Population, Eigenvalue Elasticity Analysis

Article History :

Received: 13 March 2019

Received in revised form: 29 April 2019

Accepted for publication: 03 May 2019

Published: 29 May 2019

(C)2019 Journal of the Nigerian Society of Physical Sciences. All rights reserved. Communicated by: B. J. Falaye

\section{Introduction}

Corruption is a form of dishonesty or criminal activity undertaken by a person or organization entrusted with a position of authority often to acquire illicit benefits [1]. In addition, it is the misuse of public power usually by elected politician or appointed civil servants for private gain. It is clear to every citizen that the level of corruption in the country is so high. Corruption is found in every sector of the society [2]. Be it a small or big sector, there is every possibility of observing corrupt practices when critically examined.

Forms of corruption include: taking gifts or money to speed up problem solving, extortion usually common among civil servants illegally set a fee for services or artificially create a deficit, bribery, fraud, embezzlement, falsification, forgery, nepotism, favoritism and a host of others [3]. Corruption in Nigeria wears many kinds of unattractive and dirty clothes. The situation has made so many people feel a lot of pains as the money which would have been used to reduce poverty in the country are being channeled into the pockets of a small group of persons [1]. Efforts are being made by the government of the day to reduce the rate of corruption in the country such that there will be growth and national development.

In this paper, it is intended to determine the parameter that has the greatest impact on the mathematical model of corruption which incorporates immune class and jailed class on the transmission dynamics of corruption in a certain population of the society. Therefore, the study of the transmission dynamics of an SICJR model is presented. The parameter is determined using the eigenvalue elasticity and sensitivity analyses [4] and this parameter will assist the government to reduce the rate of corrupt practices in the society. 


\section{Mathematical Formulation}

In order to formulate the mathematical model of corruption, the total population is divided into five compartments namely: Susceptible Class (S), Immune Class (I), Corrupt Class (C), Jailed Class (J) and the Reformed Class (R) with the following definitions:

a) Susceptible Class: This class consists of individuals who have never been involved in any corrupt practices that will have harmful effects on the countrys national growth and development but likely vulnerable to being infected by the corrupt practices in the society.

b) Immune Class: This class consists of individuals who can never be involved in corrupt practices irrespective of the circumstances around them.

c) Corrupt Class: This class consists of individuals who are often involved in corrupt practices and are capable of influencing the susceptible and immune individuals to become corrupt.

d) Jailed Class: This class involves individuals who have been convicted or punished of corrupt practices and imprisoned for a specific period of time during which he/she cannot be involved in any corrupt acts and cannot influence others during the imprisonment.

e) Reformed Class: This class consists of the ex-convicts who have been reformed while serving their jailed term and can become susceptible to corruption after a while.

The susceptible class is generated from daily recruitment of individuals born into homes with good moral standards and can likely be vulnerable to being infected by the corrupt practices at a rate $\theta \beta$ while the immune class are those who has moral standards from their homes and can never become corrupt at a rate $(1-\theta) \beta$ [5]. Susceptible individuals acquire corruption infection from corrupt individuals and become corrupt at a rate $\alpha$ while the corrupt individuals are jailed at a rate $\delta$. Corrupt and jailed individuals become reformed in the reformed class while serving their jail terms at rates $\tau$ and $\rho$ respectively. Individuals in the reformed class become susceptible after a while at a rate $\omega$ while susceptible individuals prone to corruption become immune at a rate $v$ due to moral and religious beliefs as well as public enlightenment campaign. All the classes are subjected to natural death at a rate $\mu$.

The above explanations result in the system of non-linear ordinary differential equations given as follows;

$$
\begin{gathered}
S^{\prime}(t)=\theta \beta-\frac{\alpha S(t) C(t)}{N(t)}-(\mu+v) S(t)+\omega R(t) \\
I^{\prime}(t)=(1-\theta) \beta+\nu S(t)-(\mu+\gamma) I(t) \\
C^{\prime}(t)=\frac{\alpha S(t) C(t)}{N(t)}+\gamma I(t)-(\mu+\tau+\delta) C(t)
\end{gathered}
$$

$$
\begin{gathered}
J^{\prime}(t)=\delta C(t)-(\mu-\rho) J(t) \\
R^{\prime}(t)=I C(t)+\rho J(t)-(\mu+\omega) R(t)
\end{gathered}
$$

The fractional system $(1-5)$ is given thus i.e.

$$
\begin{gathered}
s^{\prime}(t)=\theta \beta-\alpha s(t) c(t)-(\mu+v) s(t)+\omega r(t) \\
i^{\prime}(t)=(1-\theta) \beta+v s(t)-(\mu+\gamma) i(t) \\
c^{\prime}(t)=\alpha s(t) c(t)+\gamma i(t)-(\mu+\tau+\delta) c(t) \\
j^{\prime}(t)=\delta c(t)-(\mu-\rho) j(t) \\
r^{\prime}(t)=\tau c(t)+\rho j(t)-(\mu+\omega) r(t)
\end{gathered}
$$

The corruption transmission dynamic model is shown in Figure 1

\section{Parameter with the greatest Impact}

\subsection{Eigenvalue Elasticity and Sensitivity Analysis}

Eigenvalue elasticities measure the transient response sensitivities of the model to parameters [6] and since the values of elasticities are dimensionless, they can be compared with each other. This can aid us identifying the parameters which could greatly influence the mathematical model [7].

a) Eigenvalue Sensitivity with respect to a parameter: This is defined as the partial derivative of the eigenvalue with respect to that parameter [7]. The eigenvalue sensitivity $S_{i}(i=1, \ldots, N$ and $\mathrm{N}$ is the dimension of the state vector) with respect to the $j^{\text {th }}$ parameter of the system $p_{j}(j=1,2,3,4,5,6,7)$ is given in the form;

$$
S_{i}\left(p_{j}\right)=\Delta p_{j} \rightarrow 0 \frac{\Delta \lambda_{i}}{\Delta p_{j}}=\frac{\partial \lambda_{i}}{\partial p_{j}}=I_{i}^{T} \frac{\partial J}{\partial p_{j}} r_{i}
$$

b) Eigenvalue Elasticity with respect to a parameter: This is defined as the partial derivative of the eigenvalue with respect to that parameter normalized for the size of the parameter and the size of the eigenvalue [4]. This could also be described as the product of the eigenvalue sensitivity and the ratio of the eigenvalue and parameter [6]. Thus, it is given in the form;

$$
E_{i}\left(p_{j}\right)=\Delta p_{j} \rightarrow 0 \frac{\frac{\Delta \lambda_{i}}{\lambda_{i}}}{\frac{\Delta p_{j}}{p_{j}}}=\frac{\frac{\partial \lambda_{i}}{\lambda_{i}}}{\frac{\partial p_{j}}{p_{j}}}=\frac{\partial \lambda_{i}}{\partial p_{i}} \cdot \frac{p_{j}}{\lambda_{i}}=I_{i}^{T} \frac{\partial J}{\partial p_{j}} r_{i} \cdot \frac{p_{j}}{\lambda_{i}} .
$$

With these equations (11) and (12), the eigenvalue elasticity and sensitivity with respect to a parameter can be computed using the left eigenvectors $\left(I_{i}\right)$ and the right eigenvectors $\left(r_{i}\right)$ with the partial derivatives of the linearized Jacobian matrix $(J)$ with respect to a parameter $\left(p_{j}\right)$. Because $J$ and $\frac{\partial J}{\partial p_{j}}$ can often be easily determined symbolically and because the eigenvalues can be computed for particular parameters values and points in 


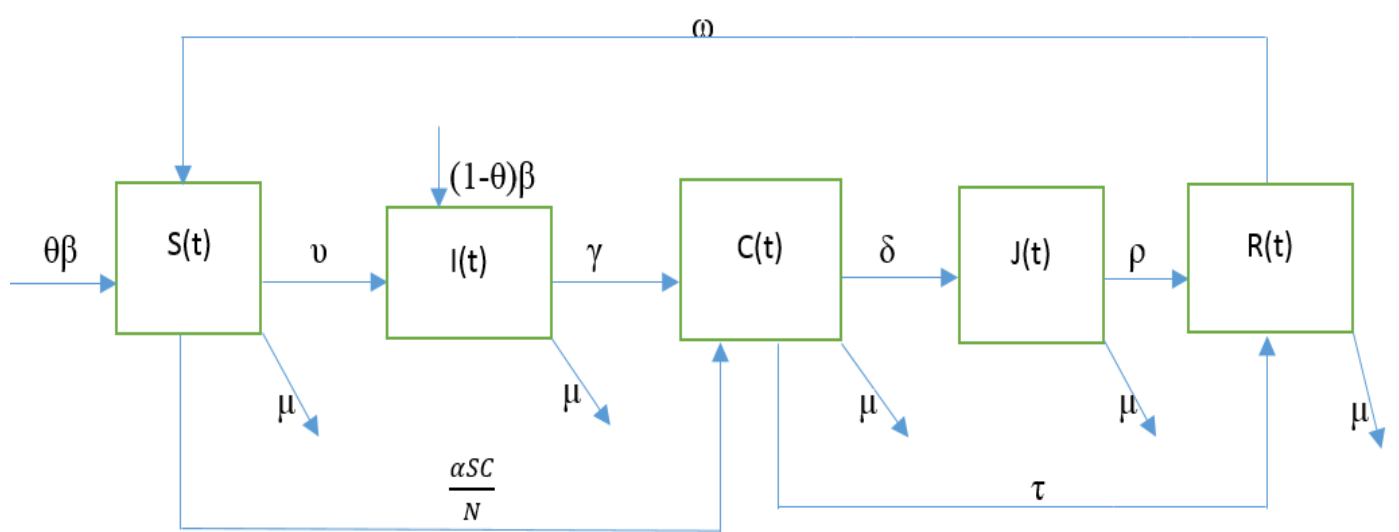

Figure 1: Diagram of the corruption transmission dynamic model.

Table 1: Definitions of Parameters used in the Mathematical Model

\begin{tabular}{lccr}
\hline \hline Parameters & Description & Values & Source \\
\hline$\theta$ & Proportion of individuals not borne immune & 0.00403 & Eguda et al. [5] \\
$\beta$ & Birth rate of individuals borne into the population & 0.042 & Eguda et al. [5] \\
$\alpha$ & Effective corruption contact rate & 0.8 & Assumed \\
$\gamma$ & Rate at which susceptible individuals become immune to corruption & 0.0004 & Eguda et al. [5] \\
$\omega$ & Rate at which reformed individuals become susceptible to corruption & 0.0021 & Eguda et al. [5] \\
$v$ & Rate at which immune individuals are susceptible to corruption & 0.143 & Eguda et al. [5] \\
$\delta$ & Rate at which prosecution and imprisonment of corrupt individuals occur & 0.0001 & Eguda et al. [5] \\
$\tau$ & Rate at which corrupt individuals become reformed due to public enlightenment & 0.001 & Assumed \\
$\rho$ & Rate at which jailed individuals become reformed & 0.0001 & Assumed \\
$\mu$ & Natural death rate & 0.0189 & Eguda et al. [5] \\
$S(t)$ & Susceptible individual class at time t. & 79,640 & Assumed \\
$I(t)$ & Immune individual class at time t. & 16,635 & Assumed \\
$C(t)$ & Corrupt individual class at time t. & 72,375 & Assumed \\
$J(t)$ & Jailed individual class at time t. & 20,695 & Assumed \\
$R(t)$ & Reformed individual class at time t. & 10,655 & Assumed \\
$N(t)$ & Fumber of Individuals in the population at time t. & 200,000 & Assumed \\
$s(t)$ & Fraction of Susceptible Individual at time t. & 0.3982 & Estimated \\
$i(t)$ & Fraction of the Immune Individual at time t. & 0.0832 & Estimated \\
$c(t)$ & Fraction of the Corrupt Individual at time t. & 0.3619 & Estimated \\
$j(t)$ & Fraction of the Jailed Individual at time t. & 0.1035 & Estimated \\
$r(t)$ & Fraction of the Reformed Individual at time $t$. & 0.0533 & Estimated
\end{tabular}

time, both eigenvalue elasticity and sensitivity with respect to a parameter can be computed without the need to either compute closed form expressions for eigenvalues nor to perform numeric differentiation [4].

Using the MATLAB software package [8], the computer program was written for the evaluation of the values of eigenvalue elasticity and sensitivity of the mathematical model given in equations (6)-(10). Definitions of parameters used in the Mathematical Model are shown in Table 1 The results obtained are shown in the Table 2 and the computer program is shown under the appendix. 
Table 2: The values of the eigenvalue sensitivity and elasticity analysis

\begin{tabular}{lccr}
\hline \hline Parameters & Definition of Parameters & Eigenvalue Sensitivity & Eigenvalue Elasticity \\
\hline$\alpha$ & Effective corruption contact rate & 1.000064625 & 1.025787253 \\
$\nu$ & Natural death rate & -1.0000 & -0.002423266 \\
$v$ & $\begin{array}{c}\text { Rate at which immune individuals are } \\
\text { susceptible to corruption }\end{array}$ & -0.0003606 & -0.000066117 \\
$\gamma$ & $\begin{array}{c}\text { Rate at which susceptible individuals } \\
\text { become immune to corruption }\end{array}$ & -0.151919916 & -0.000007791372 \\
$\omega$ & $\begin{array}{c}\text { Rate at which reformed individuals } \\
\text { become susceptible to corruption }\end{array}$ & 0.00000009464 & 0.000000002548 \\
$\tau$ & $\begin{array}{c}\text { Rate at which corrupt individuals become } \\
\text { reformed due to public enlightenment } \\
\text { Rate at which prosecution and }\end{array}$ & -1.0001404 & -0.00128233 \\
$\rho$ & imprisonment of corrupt individuals occur & -1.0001406 & -0.01283315 \\
$\rho$ & Rate at which jailed individuals become reformed & 0.00000000024938 & 0.031973813 \\
\hline
\end{tabular}

\section{Discussion and Recommendations}

Table 2 shows that the parameter alpha $(\alpha)$ which is defined as the effective corruption contact rate has the highest eigenvalue elasticity and eigenvalue sensitivity values. This means that the parameter has the greatest impact on the formulated mathematical model of the corruption model. This is to show that the parameter should be thoroughly investigated and worked upon as a possible policy lever such that the rate of corrupt transmission will be reduced to the barest minimum by the public officials especially all the government agencies that deal with corrupt practices (EFCC, ICPC, etc). In order to reduce the rate of effective corruption contact among the populace, the following recommendations are necessary:

1. Self satisfaction: this means that an average human being should be contented with whatever he or she has. When the leaders are satisfied with the salary they are being paid and use them in the right way, the issue of embezzlement and money laundering will be stopped. Managers, civil servants, etc. who are satisfied with what they are paid will not have time to indulge in corruption to make more money.

2. Creating strong and efficient anti corruption institutions: another arsenal to win the fight against corruption and the reduce the effective rate of corruption contact is for the government to establish more anti corruption agencies in addition to the already established ones. These agencies will work independently with the government to ensure transparency. Anyone who is caught in corrupt practices by any of the agencies should experience the consequences decided by the anti corruption agencies.

3. Equal treatment: treating any corruption offender equally will help to reduce the rate of effective corruption contact. Nobody is above the law and any who acts contrary to the rule of law should be given the adequate punishment that he or she deserves.

\section{Conclusion}

In this paper, a five compartmental model has been presented to gain insight on the parameter that has the greatest impact on the mathematical model of the transmission dynamics of corruption. The result from the research has shown that the rate of effective corruption contact among the populace has the greatest impact. With this result, when necessary and adequate measures are taken by the government, it is enough and expedient to reduce to the barest minimum the spread of corruption among the populace.

\section{References}

[1] U. M. P. Okwuagbala, "Corruption in Nigeria: Review, Causes and Solutions", 2018

[2] O. A. Oyinlola, Corruption Eradication in Nigeria: An Appraisal, Library Philosophy and Practice, 2011.

[3] S. Abdulrahman, "Stability Analysis of the transmission dynamics and control of corruption", The Pacific Journal of Science and Technology, 15 (2014) 99.

[4] A. O. Binuyo, "Eigenvalue Elasticity Analysis of an SEIRC epidemic model for an infectious disease", Elixir Electrical Engineering Online Journal 53 (2012) 11928.

[5] F. Y. Eguda, F. Oguntolu \& T. Ashezua, "Understanding the Dynamics of Corruption using Mathematical Modelling Approach", International Journal of Innovative Science, Engineering \& Technology, 4 (2017) 190.

[6] B. Guneralp, Progress in Eigenvalue Elasticity Analysis as a Coherent Loop Dominance Analysis Tool, In Proc. the 23rd International Conference of the system dynamics Society, Boston, MA, 2005.

[7] Q. Zhang, Application and Evaluation of Local and Global Analysis for Dynamic Models of Infectious Disease Spread, Master's thesis project, Department of Computer Science, University of Saskatchewan, Saskatoon, 2008.

[8] R. H. Brian, A guide to MATLAB for beginners and experienced users, Cambridge University Press, webnote, 2001. 
Appendix: Eigenvalue Elasticity and Sensitivity Analysis Program

syms mu nu alpha omega gamma

tau delta rho; $\mathrm{mu}=0.0189 \mathrm{nu}=0.143$

alpha $=0.8$ omega $=0.0021$ gamma $=0.0004$

tau $=0.001$ delta $=0.0001$ rho $=0.0001$

mat31 = $[-(m u+n u) 0-a l p h a ~ 0$ omega;

nu - (mu+gamma) 000 ;

0 gamma alpha-(mu+tau+delta) 00 ;

0 delta - (mu+rho) 0 ;

0 o tau rho -(mu+omega)]

[wmat31, dmat31] = eig (mat31)

$\operatorname{vmat} 31=\operatorname{conj}($ inv $($ wmat31) $)$

lambdiag $=\operatorname{diag}(\operatorname{dmat31})$

lambvec $=\operatorname{sort}(\operatorname{abs}($ lambdiag $))$

$\operatorname{dim}=\operatorname{size}($ lambvec, 1$)$

lambda1 $=\operatorname{lambvec}(\mathrm{dim})$

PD_mu $=\operatorname{diff}(\operatorname{mat} 31, \mathrm{mu})$

PD_nu $=\operatorname{diff}(\operatorname{mat} 31, \mathrm{nu})$

PD_alpha $=\operatorname{diff}(\operatorname{mat} 31$, alpha $)$

PD_omega $=\operatorname{diff}($ mat31, omega $)$

$P D_{\text {_gamma }}=\operatorname{diff}(\operatorname{mat} 31$, gamma $)$

PD_tau $=\operatorname{diff}(\operatorname{mat} 31$, tau $)$

PD_delta $=\operatorname{diff}(\operatorname{mat} 31$, delta $)$

PD_rho $=\operatorname{diff}(\operatorname{mat} 31$, rho $)$

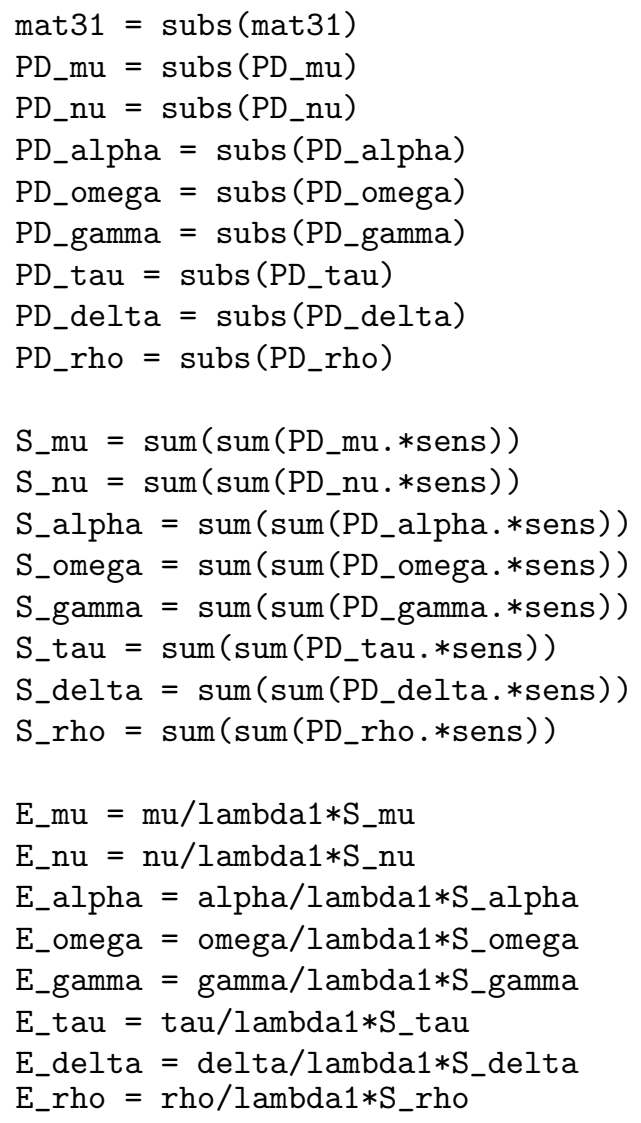

